\title{
THE EFFICACY OF VESTIBULAR REHABILITATION IN PATIENTS WITH CHRONIC UNILATERAL VESTIBULAR DYSFUNCTION
}

\author{
WOJCIECH SMÓŁKA ${ }^{1}$, KATARZYNA SMÓŁKA르, JAROSŁAW MARKOWSKI ${ }^{1}$, JAN PILCH ${ }^{1}$, \\ AGNIESZKA PIOTROWSKA-SEWERYN ${ }^{1}$, and ANNA ZWIERZCHOWSKA ${ }^{3}$ \\ ${ }^{1}$ Medical University of Silesia, Katowice, Poland \\ Clinical Department of Otolaryngology \\ ${ }^{2}$ Rehabilitation and Educational Center for Disabled Children in Jaworzno, Jaworzno, Poland \\ ${ }^{3}$ Jerzy Kukuczka Academy of Physical Education in Katowice, Katowice, Poland \\ Institute of Sport Science
}

\begin{abstract}
Objectives: Vestibular rehabilitation leads to a gradual diminution of the subjective and objective symptoms that accompany the vestibular disorders. The aim of the study was to compare the impact of 2 different types of vestibular rehabilitation on vestibular compensation in patients with chronic unilateral vestibular dysfunction. Material and Methods: The study was conducted on a group of 58 subjects (43 females and 15 males) aged 40-64 years, who presented with chronic unilateral vestibular dysfunction and were hospitalized. The patients were randomly assigned to either of the 2 groups established. The study was conducted in a 6-week period. Group 1 consisted of patients who underwent customized group vestibular rehabilitation in an outpatient setting. The program was performed once a week for $1 \mathrm{~h} 30 \mathrm{~min}$, under the supervision of a physiotherapist and a physiatrist. Group 2 was instructed to perform Cawthorne-Cooksey exercises and simple balance exercises twice a day for 15 min. Results: An improvement in the outcomes of the Dynamic Gait Index as well as the Berg Balance Scale was statistically significant for group 1. The time for fulfilling the task in the Timed Up and Go Test improved in both groups $(\mathrm{p}<0.05)$. The subjective estimation of the symptoms evaluated with the use of the Dizziness Handicap Inventory and the Visual Analogue Scale revealed a statistically significant improvement in both groups, yet it was higher in group 1. Conclusions: The compensation achieved after 6 weeks of the customized, supervised outpatient rehabilitation program in group 1 was superior to the results of the home-based unsupervised Cawthorne-Cooksey and balance exercises. Int J Occup Med Environ Health. 2020;33(3):273-82
\end{abstract}

Key words:

rehabilitation, balance disorders, working adults, vestibular, exercises, practical experience

\section{INTRODUCTION}

Vertigo, dizziness and disequilibrium are diagnosed by general practitioners (GPs) in 5-7\% of patients and by ear, nose and throat (ENT) specialists in about 10-12\% of cases. The disorder is most common in elderly people, especially in the sixth and seventh decade [1]. The symptoms depend on the origin of the disease; therefore, ves- tibular and non-vestibular causes of dizziness/vertigo can be distinguished [2]. In the case of a central vestibular disorder (a dysfunction of $\geq 1$ parts of the central nervous system that help process balance and spatial information), the patients suffer from dizziness, i.e., a non-specific sensation of spinning, whirling, unsteadiness, imbalance when walking or standing, and spatial disorientation. The onset

Funding: this study was supported by the Medical University of Silesia and the Jerzy Kukuczka Academy Physical Education in Katowice.

Received: February 8, 2018. Accepted: January 9, 2020.

Corresponding author: Anna Zwierzchowska, Jerzy Kukuczka Academy of Physical Education, Institute of Sport Science, Mikołowska 72a, 40-066 Katowice, Poland (e-mail: a.zwierzchowska@awf.katowice.pl). 
of these symptoms is difficult to be precisely estimated; their intensity is changeable and mostly not influenced by head movements. Other neurological disorders, such as consciousness disorders, headache or epilepsy, might accompany the above symptoms [2].

A peripheral vestibular disorder (a dysfunction of the balance organs of the inner ear) is characteristic of vertigo, i.e., a feeling of whirling with a sudden onset, a gradual diminution and a minute-to-hour duration. The symptoms increase with head movements or eye closure. Vegetative symptoms such as nausea, vomiting, sweating, or an increased heart rate are commonly observed in this type of vestibular dysfunction. The diseases, in which a vestibular disorder is present, are as follows:

- peripheral neuritis,

- diseases of the inner ear of an unknown origin, e.g., Ménière's disease,

- vestibular otosclerosis,

- benign paroxysmal positional vertigo (BPPV),

- traumas of the inner ear,

- neoplasms of the inner ear,

- systemic diseases,

- vascular disorder affecting the inner ear.

As many as 30-50\% of all cases of vertigo/dizziness are due to peripheral vestibular dysfunction [3-5]. Studies have shown that vertigo, disequilibrium and nausea might subside spontaneously after 3-4 months from the symptoms onset thanks to processes of central compensation in the region of vestibular nuclei and reticular formation [6-10]. However, in order to compensate for vestibular dysfunction, it is essential to stimulate vision or proprioception. Vestibular rehabilitation leads to a gradual diminution of the subjective and objective symptoms that accompany the vestibular disorder [10-12].

The medications taken, the level of sensorial stimulation, the rehabilitation onset and its duration, the intensity of symptoms, the location of damage to the vestibular organ, as well as the patient's age and psychological fac- tors may influence the efficacy of vestibular rehabilitation. A static compensation, i.e., the lack of vertigo when there is no movement, can be easily achieved. However, a total central compensation is achieved when there are no symptoms during daily activities. In certain cases, due to the lack of physical activity, and especially the lack of head movements initiating vertigo, a total central compensation cannot be obtained. According to the modeling theory, if information is perceived by all senses (vestibular, visual and proprioception) as expected, the equilibrium is sustained. In the case of damage to the vestibular organ, there is no balance and each movement causes vertigo as a result of false image perception (danger-induced stimuli). However, a systematically repeated stimulus that is no longer associated with danger creates a new equivalent image in the central nervous system, which is perceived as acceptable. The above process inhibits further reactions, i.e., the occurrence of vertigo [13].

The goals of vestibular rehabilitation cover: an improvement of visual acuity when moving, a decrease in nystagmus, an improvement of postural control, a diminution of vertigo, an improvement of the general fitness and physical activity, the prophylaxis of sudden falls, and an improvement of the quality of life, especially as regards its social aspects [1,13-16].

The aim of the study was to compare the impact of 2 different types of vestibular rehabilitation on vestibular compensation in patients with chronic unilateral vestibular dysfunction.

\section{MATERIAL AND METHODS \\ Participants}

The study was conducted on a group of 58 subjects (43 females and 15 males) aged 40-64 years, who were referred to the Clinical Department of Otolaryngology, Medical University of Silesia in Katowice, in 2008-2011 with the symptoms of vertigo and the loss of balance, commonly resulting in falls, in whom chronic unilateral vestibular dysfunction 
was diagnosed on the basis of anamnesis, clinical examinations and the results of electronystagmography with caloric tests. Each patient additionally underwent pure tone audiometry, impedance audiometry and vestibular evoked myogenic potentials. The character and duration of the clinical symptoms, the asymmetry of caloric response confirming the diagnosis of chronic unilateral vestibular dysfunction, and the patient's written consent acted as the inclusion criteria. The exclusion criteria were as follows:

- central migraine,

- a neoplastic disease,

- BPPV,

- Ménière's disease,

- perilymphatic fistula,

- symptoms persisting for $<4$ months,

- other disturbances that would preclude rehabilitation according to the appropriate protocol, i.e., gait dysfunction, neurological disorders, musculoskeletal system diseases, or vascular diseases (>NYHA II - New York Heart Association Classification Class II), or myocardial insufficiency.

Group 1 consisted of 19 females and 8 males, whose mean age was 53.7 years. Group 2 included 24 females and 7 males, whose mean age was 51.94 years. The characteristics of both these groups is presented in Table 1 .

On physical examination, vertigo and disequilibrium were revealed in all patients. The mean duration of the symptoms ranged 4-42 months (mean duration: 16.59 months) in group 1, and 5-45 months (mean duration: 21.90 months) in group 2.

\section{Procedures}

The patients were randomly assigned to either of the 2 groups established. The study was conducted in a 6-week period. Group 1 consisted of patients who underwent customized group vestibular rehabilitation in an outpatient setting. The program was performed once a week for $1 \mathrm{~h} 30 \mathrm{~min}$, under the supervision of a physiotherapist and a physiatrist.
Each session consisted of general conditioning exercises, balance, postural, gait stability and spatial orientation training, and head and goal directed eye movement (gaze stability) exercises, which were conducted in small groups. Additionally, each patient performed visual feedback exercises on the ALFA stabilometric platform (AC International East). ALFA is a modern stabilometric platform that allows both the balance assessment and training in neurological and orthopedic patients. Training on the platform aims at stimulating musculoskeletal and nervous system elements responsible, i.a., for balance control. The ALFA stabilometric platform cooperates with software so that the patient is aware of the challenges and progress in rehabilitation, which increases his/her motivation. The capabilities of the platform include the evaluation of static parameters involved in maintaining the balance on a stable surface, the analysis of the centre of pressure (COP) during testing and training, templates for testing and training programs, and the ability to create one's own exercises with integrated real-time biofeedback, the objectification of the rehabilitation process, and the adaptation of the exercise level of difficulty to the current needs of the patient.

Group 2 was instructed to perform Cawthorne-Cooksey exercises and simple balance exercises $[17,18]$ twice a day for $15 \mathrm{~min}$. The overall compliance was evaluated using diaries filled in by the patients every day. The frequency and duration of exercises was also recorded.

The outcomes were measured at the baseline and final (6 weeks after the onset of rehabilitation) visits. All patients were examined and the following parameters were assessed.

1. Balance assessment on the ALFA stabilometric platform - a stabilometric test with one's eyes open and closed. The following parameters were analyzed: distance (path length) for COP (with one's eyes open and closed), surface area of the stabilogram (with one's eyes open and closed), the ratio of the surface area of the stabilogram with one's eyes closed over the surface area of 
Table 1. Dynamic test, distance gained and the distance ratio for 2 groups of patients $(\mathrm{N}=58)$ in the study on the impact of vestibular rehabilitation on patients with chronic unilateral vestibular dysfunction in 2008-2011, Katowice, Poland

\begin{tabular}{lcccc}
\hline \multirow{2}{*}{ Distance gained and distance ratio } & \multicolumn{4}{c}{ Dynamic test results } \\
\cline { 2 - 5 } & \multicolumn{2}{c}{ group 1 } & \multicolumn{2}{c}{ group 2 } \\
\cline { 2 - 5 } Distance [cm] & before the test & after the test & before the test & after the test \\
M & & & & \\
SD & 693.1 & 474.3 & 633.9 & 625.8 \\
SEM & 175.3 & 150.4 & 209.7 & 220.4 \\
Me & 33.7 & 28.9 & 37.7 & 39.6 \\
lower quartile & 699.4 & 457.9 & 558.5 & 663.8 \\
upper quartile & 517.5 & 358.7 & 466.0 & 406.0 \\
min. & 771.6 & 530.8 & 826.0 & 763.8 \\
max & 480.0 & 307.3 & 365.0 & 345.0 \\
Wilcoxon's test & 1169.1 & 956.8 & 1024.9 & 977.5 \\
Distance ratio: reached/minimal [\%] & & $\mathrm{p}=0.0001$ & & $\mathrm{p}=0.0152$ \\
M & & & & \\
SD & 404.7 & 276.6 & 368.2 & 365.4 \\
SEM & 103.5 & 84.0 & 125.1 & 130.3 \\
Me & 19.9 & 16.2 & 22.5 & 23.4 \\
lower quartile & 411.0 & 268.0 & 328.0 & 390.0 \\
upper quartile & 298.0 & 210.0 & 252.0 & 240.0 \\
min. & 453.0 & 311.0 & 485.0 & 448.0 \\
max & 281.0 & 180.0 & 216.0 & 202.0 \\
Wilcoxon's test & 684.0 & 524.0 & 586.0 & 575.0 \\
\hline
\end{tabular}

Group 1 - patients $(\mathrm{N}=27)$ who underwent customized group vestibular rehabilitation in an outpatient setting; group 2 - patients $(\mathrm{N}=31)$ who performed Cawthorne-Cooksey exercises and simple balance exercises twice a day for $15 \mathrm{~min}$.

the stabilogram with one's eyes open, and the ratio of distance (path length) for COP with one's eyes closed over the path length for COP with one's eyes open.

2. Limit of Stability (LOS) - a test assessing the patient's ability to control his/her COP movement by tilting his/her body in different directions in order to achieve the maximum amount of targets on the screen in the shortest time possible, using biofeedback. Each task is performed on an auditory signal. The parameters analyzed include the path length for COP, the ratio of the path in total to the minimal path, and the time needed to reach targets in 4 quadrants of the screen.

3. The Dynamic Gait Index (DGI) - a test composed of 8 exercises, each scored 0 (severe impairment) to 3 pts (the highest level of functioning); the maximum score: 24 pts; a score of $<19$ pts - predictive of falls.

4. The Berg Balance Scale (BBS) - a balance assessment test composed of 14 exercises, e.g., the skills of sitting, turning, and standing on 1 leg; each task is scored 0 (the lowest level of functioning) to 4 pts (the highest 
level of functioning); the maximum score: 56 pts; 4156 pts - a low fall risk; 21-40 pts - a medium fall risk; and $0-20$ pts - a high fall risk.

5. The Timed Up and Go Test - a test during which the patient sits on a chair, stands up, walks $3 \mathrm{~m}$ forward, turns round, goes back towards the chair and sits down; the test results correspond with walking velocity, balance and general agility; a score of $\geq 14 \mathrm{~s}$ is predictive of falls.

6. The Dizziness Handicap Inventory (DHI) as proposed by Jacobson and Newman (1990).

7. Vertigo evaluation with the Visual Analogue Scale (VAS) - a 10-pt scale of self-assessment of the symptoms level.

The study was conducted according approval No. KNW/ 0022/KB1/148/11 issued by the Bioethics Committee of the Medical University of Silesia.

\section{Statistical analysis}

Statistical analysis was performed with the use of Statistica 7.1 PL. A p-value of $<0.05$ was regarded as statistically significant. The distribution was evaluated with the ShapiroWilk test. A non-parametric Mann-Whitney U test and tStudent test were used to assess differences for unpaired variables between the 2 groups, and Wilxocon's test within the groups.

\section{RESULTS}

Subjectively, a decrease in the symptoms of nystagmus and vertigo were reported by all patients in both groups, yet it was significantly higher in group 1 .

A decrease in the distance for COP and the surface area of the stabilogram with one's eyes open and closed was statistically significant in group 1 . Yet, in group 2 statistical significance was obtained only for the surface area of the stabilogram with one's eyes closed. Both ratios for group 1, and the ratio for the surface area for group 2, decreased significantly (Figures 1 and 2).

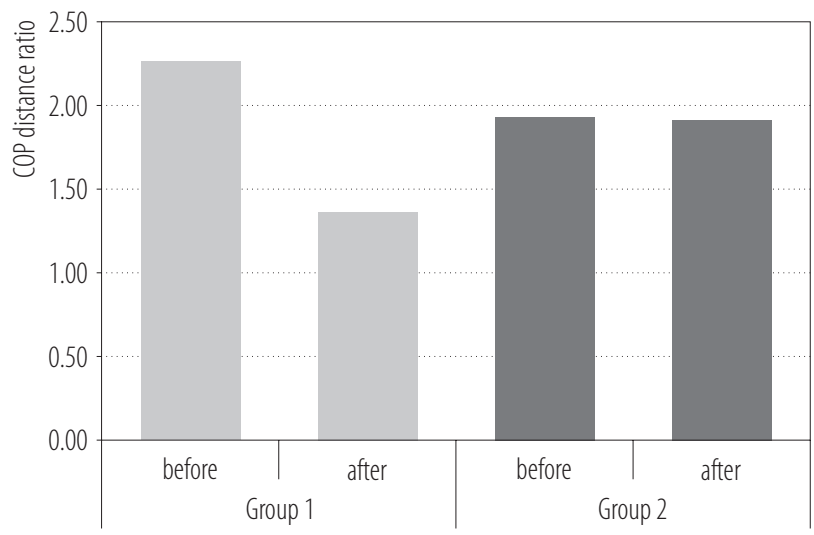

Figure 1. Ratio of the distance for the centre of pressure (COP) with one's eyes closed over the distance for COP with one's eyes open in 2 groups of patients $(\mathrm{N}=58)$ in the study on the impact of vestibular rehabilitation on patients with chronic unilateral vestibular dysfunction

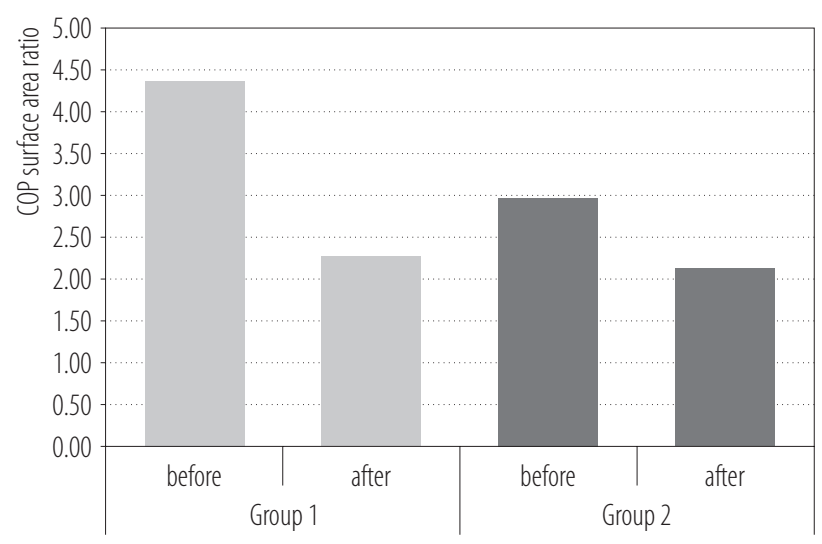

Figure 2. Ratio of the surface area for the centre of pressure (COP) with one's eyes closed over the distance for COP with one's eyes open in 2 groups of patients $(\mathrm{N}=58)$ in the study on the impact of vestibular rehabilitation on patients with chronic unilateral vestibular dysfunction

An analysis of the results of the staggering velocity in axial (axis Y) and frontal planes (axis X) at the baseline and final visits revealed that a decrease in the velocity with one's eyes open and closed could be observed only in group 1 . A statistically significant improvement $(p=0.0001)$ in all parameters evaluated before and after rehabilitation, i.e., the path length of COP, the ratio of distance gained to the minimal distance, and the total time for fulfilling 
the task, were observed in the test with the use of the ALFA stabilometric platform in group 1 (Table 1).

The results were significant in group 2 only in the case of the distance for COP and the total time for fulfilling the task.

Also, the time for fulfilling the task was assessed for all quadrants, i.e., frontal right (F-R), frontal left (F-L), posterior right $(\mathrm{P}-\mathrm{R})$ and posterior left $(\mathrm{P}-\mathrm{L})$. The result of $>60 \mathrm{~s}$ was regarded as the one in which the patient was unable to fulfill the task. A statistically significant improvement in all quadrants could be observed in the case of group 1 .

An improvement in the outcomes of DGI as well as the Berg Balance Scale was statistically significant for group 1. The time for fulfilling the task in the Timed Up and Go Test improved in both groups $(\mathrm{p}<0.05)$. The subjective estimation of the symptoms evaluated with the use of the Dizziness Handicap Inventory and VAS revealed a statistically significant improvement in both groups, yet it was higher in group 1.

\section{DISCUSSION}

Vestibular rehabilitation is a gold standard in the case of chronic unilateral vestibular dysfunction. Since the introduction of the Cawthorne-Cooksey rehabilitation protocol in the 1940s, the methods have evaluated gradually $[17,18]$. A great role of vision and proprioception was emphasized in the 1970s, especially by Zee, the pioneer of the method [19]. The first prospective and randomized studies conducted in the 1990s revealed a beneficial effect of vestibular rehabilitation on the function of vestibular organs [20]. Since then, more and more authors have focused on individual rehabilitation protocols that would fit patient's needs and abilities [14,21,22]. Horak et al. [9], Han et al. [14], and Eleftheriadou et al. [23] have claimed that exercises with a gradually increasing level of difficulty could be performed both at patient's home, under the constant supervision of a physiatrist, and at a rehabilitation centre.
The rehabilitation program presented in this study was based on the experience gathered by the authors of this study and by other authors in respect to the units of regular Polish rehabilitation centers, so that the results would reflect clinical implications. It was difficult to compare these results with other studies, due to the fact that the material was not homogeneous. For instance, several researchers included in their studies groups of patients with both peripheral and central dysfunctions of the vestibular organ [23,24], BPPV [25-29], bilateral vestibular dysfunction [30], or postoperative patients due to neurinomas of the vestibulocochlear nerve $[31,32]$. Some studies presented results for groups of patients with vertigo and/or disequilibrium with no respect to the etiology of the symptoms $[9,33]$.

The study group involved in this study was carefully selected. The exclusion criteria covered BPPV (as it has been found that repositional maneuvers in these diseases are much more effective than in other disorders) [26,34], perilymph fistula (as its main treatment is a surgical procedure) and Ménière's disease (due to its paroxysmal character) [2]. Also, differences in the onset of rehabilitation after the first symptoms could be observed. Some authors assessed the influence of vestibular rehabilitation in the acute phase of the disease [35], while others several months or years from the first symptoms [36,37]. Patients who were included in this study had suffered from vertigo/ disequilibrium for $\geq 4$ months. This minimum period inclusion criterion was set in order to avoid the influence of spontaneous compensation. Similarly, various methods of assessing the rehabilitation outcome were used, e.g., the evaluation of adaptation exercises in respect of dynamic visual acuity (DVA) [38], posturography, and clinical tests with an additional assessment of depression and anxiety levels [39]. Teggi et al. [40] evaluated the effect of vestibular rehabilitation with the use of DGI, VAS and DHI questionnaire.

It is the authors' firm belief that the methods introduced to this study were sufficient, as both the objective and 
subjective aspects of the vestibular rehabilitation outcome were assessed. Hillier and McDonnell [35] performed a literature review of the common methods of physiotherapy in unilateral vestibular dysfunction. They found that 27 authors were able to prove the safety and efficacy of vestibular rehabilitation, and its severalmonth resistance. However, they did not indicate 1 particular type of a rehabilitation method as superior to others.

Several scientists have compared the rehabilitation protocols in the discussed disease. Cohen et al. [37] have revealed that 4-week exercises, which were based on slow movements of the head (group 1), fast movements of the head (group 2) and fast movements of the head with a contact with the investigator once a week (group 3), resulted in a decrease in vertigo and an improvement in the social functioning in all groups. They emphasized that even a slow motion of the head was likely to lead to habituation. The comparison of the above results with the outcomes achieved in this study seems impossible due to the fact that only subjective assessment was presented by Cohen et al., and there was no evaluation of the equilibrium.

Kammerlind et al. [36] presented a study of 54 subjects who underwent a program of visual-motional exercises on a home-based protocol, in comparison with a group in which an additional kinesiotherapy was performed in a rehabilitation centre. The outcomes were estimated after several days from the onset of the disease. Therefore, the authors of this article believe that a great disadvantage of the study is that it remains uncertain whether the results were the effect of the described rehabilitation or spontaneous compensation.

The results presented by Zimbelman et al. [41], who revealed a statistically significant improvement in the DHI scale in the group of patients who underwent complex rehabilitation, correspond positively with this study. Yet, in this research, an improvement was observed in both groups. This discrepancy may have resulted from the different material being used by Zimbelman et al., which included subjects with Ménière's disease and BPPV.

Pośpiech [13] underlined the superiority of combined motional and optokinetic training. Szturm et al. [42], instead, emphasized the necessity for visual fixation and better results for a customized, supervised outpatient rehabilitation program. Similar conclusions were presented by Pavlou et al. [43]. Both the above studies and the results obtained in this study provide the basis to claim that a combined outpatient rehabilitation protocol is superior to a homebased program. The authors believe that a patient who trains under the supervision of a physiatrist is ready to perform more difficult tasks, is less worried about sudden falls, is more precise, and sticks to the rhythm of the exercises. Moreover, group therapy is not without a positive impact, as it favors rivalry and stimulates motivation. It is worth reminding that, according to Pośpiech, the patient's psychological attitude is the basic factor of effective kinesiotherapy [13].

As far as new technologies are concerned, virtual reality was introduced in patient training on a running track by Mulavara et al. [44]. Pavlou et al. [45] evaluated the influence of a virtual crowd on the vestibular rehabilitation effect, achieving promising results. Wall et al. [46] and Dozza et al. [47] assessed the advantages of a vibrotactile array attached to the torso for somatosensory stimulation, which indicated a decline in bodily functions through vibrations. However, the authors claim that above methods might be efficient in the case of acute disequilibrium.

Stabilometric platforms seem common items for gait rehabilitation, especially when biofeedback is applied. Although it was impossible to assess this method as a separate one, the authors observed that the patients were willing to perform exercises on such a platform. Similar conclusions were presented by Cakrt et al. [48], who showed an improvement in stability thanks to vestibular rehabilitation combined with visual biofeedback. 
The authors believe that customized vestibular rehabilitation under the supervision of a physiatrist (a medical doctor specializing in rehabilitation) and an ENT specialist is a gold standard in the treatment of unilateral vestibular dysfunction. New technologies with the use of biofeedback improve training outcomes and enable the monitoring of therapeutic processes.

\section{CONCLUSIONS}

Compensation achieved after 6 weeks of a customized, supervised outpatient rehabilitation program in group 1 is superior to the results achieved with home-based unsupervised Cawthorne-Cooksey and balance exercises.

The results of the Berg Balance Scale and DGI, as well as VAS, are the most significant parameters to assess when it comes to the efficacy of vestibular rehabilitation in patients with chronic unilateral vestibular dysfunction.

It is essential to improve the co-operation of ENT specialists, physiatrists and physiotherapists in order to achieve better results of vestibular rehabilitation in patients with chronic unilateral vestibular dysfunction. This might result in a higher quality of life and, in many cases, in an opportunity for the patients to return to their workplace.

\section{ACKNOWLEDGMENTS}

The authors are grateful to the participants for their time and effort.

\section{REFERENCES}

1. Yardley L, Luxon L. Treating dizziness with vestibular rehabilitation. BMJ. 1994;308(14):1252-3, https://doi.org/10.1136/ bmj.308.6939.1252.

2. Bisdorff A, von Brevern M, Lempert T, Newman-Toker DE. Classification of vestibular symptoms: towards an international classification of vestibular disorders. J Vestib Res. 2009;19(1-2):1-13, https://doi.org/10.3233/VES-2009-0343.

3. Neuhauser HK, von Brevern M, Radtke A, Lezius F, Feldmann M, Ziese T, et al. Epidemiology of vestibular vertigo: a neurotologic survey of the general population. Neurology. 2005;65(6):898-904, https://doi.org/10.1212/01.wnl.0000 175987.59991.3d.

4. Timothy C. Hain, MD. Dizziness, imbalance and hearing disorders [Internet]. Chicago: Chicago Dizziness and Hearing; 2006 [cited 2018 Jan 5]. Available from: https://dizziness-and-balance.com/disorders/index.html.

5. Kroenke K, Hoffman RM, Einstadter D. How common are various causes of dizziness? A critical review. South Med J. 2000;93(2):160-7.

6. Brandt T. Vestibular disorders in (frontal) roll plane. [In:] Vertigo. Its multisensory syndromes. Springer; 2000. p. 175-95.

7. Abatzides GJ, Kitsios A. The role of rehabilitation in the treatment of balance disorders. J Back Musculoskelet Rehab. 1999;12(2):101-12.

8. Cohen H. Disability and rehabilitation in the dizzy patient. Curr Opin Neurol. 2006;19(1):49-54, https://doi.org/10.1097/ 01.wco.0000194373.08203.33.

9. Horak FB, Jones-Rycewicz C, Owen Black F. Effects of vestibular rehabilitation on dizziness and imbalance. Otolaryngol Head Neck Surg. 1992;106(2):175-80.

10. Bień S. [Kliniczne aspekty kompensacji przedsionkowej]. Bibl Prospera Ménière’a. 1997;1(1). Polish.

11. Pośpiech L. Rehabilitacja następstw narządów przedsionkowych. In: Janczewski G, Latkowski B, editors. Otoneurologia. Warszawa: Bel Corp Scient; 1998. p. 503-14. Polish.

12. Lacour M, Dutheil S, Tighilet B, Lopez C, Boel C. Tell me your vestibular deficit, and I'll tell you how you'll compensate. Ann NY Acad Sci. 2009;1164:268-78, https://doi. org/10.1111/j.1749-6632.2008.03731.x.

13. Pośpiech L. [Kinezyterapia zawrotów głowy i zaburzeń równowagi w uszkodzeniu narządu przedsionkowego (praca habilitacyjna)]. Akademia Medyczna we Wrocławiu; 1989. Polish.

14. Han BI, Song HS, Kim JS. Vestibular rehabilitation therapy: review of indications, mechanisms, and key exercises. J Clin Neurol. 2011;7(4):184-96, https://doi.org/10.3988/jcn. 2011.7.4.184. 
15. Herdman SJ. Advances in the treatment of vestibular disorders. Phys Ther. 1997;77(6):602-18. https://doi.org/10.1093/ $\mathrm{ptj} / 77.6 .602$.

16. Macias JD, Massingale S, Gerkin RD. Efficacy of vestibular rehabilitation therapy in reducing falls. Otolaryngol Head Neck Surg. 2005;133(3):323-5, https://doi.org/10.1016/j.otohns. 2005.04.024.

17. Jacobson GP, Newman CW. The Development of the Dizziness Handicap Inventory. Arch Otolaryngol Head Neck Surg. 1990;116(4):424-7, https://doi.org/10.1001/archotol.1990. 01870040046011.

18. Cawthorne T. Vestibular injuries. Proc R Soc Med. 1946; 39(5):270-2.

19. Cooksey FS. Rehabilitation in vestibular injuries. Proc R Soc Med. 1946;39(50):273-8.

20. Zee DS. Treatment of vertigo. In: Johnson RT, editor. Current therapy in neurologic diseases. Philadelphia: BC Decker; 1985. p. 8-13.

21. Krebs DE, Gill-Body KM, Riley PO, Parker SW. DoubleBlind, Placebo-Controlled Trial of Rehabilitation for Bilateral Vestibular Hypofunction: Preliminary Report. Otolaryngol Head Neck Surg. 1993;109(4):735-41, https:/doi. org/10.1177/019459989310900417.

22. Hooray FB. Postural compensation for vestibular loss and implications for rehabilitation. Restor Neurol Neurosci. 2010;28(1):57-68, https://doi.org/10.3233/RNN-2010-0515.

23. Eleftheriadou A, Skalidi N, Velegrakis A. Vestibular rehabilitation strategies and factors that affect the outcome. Eur Arch Otorhinolaryngol. 2012;269(11):2309-16, https://doi. org/10.1007/s00405-012-2019-2.

24. Yardley L, Beech S, Zander L, Evans T, Weinman J. A randomized controlled trial of exercise therapy for dizziness and vertigo in primary care. Br J Gen Pract. 1998;48(429): 1136-40.

25. Badke MB, Shea TA, Miedaner JA, Grove CR. Outcomes after rehabilitation for adults with balance dysfunction. Arch Phys Med Rehabil. 2004;85(2):227-833, https://doi. org/10.1016/j.apmr.2003.06.006.
26. Chang W, Yang Y, Hsu L, Chern C, Wang R. Balance improvement in patients with benign paroxysmal positional vertigo. Clin Rehab. 2008;22(4):338-47, https://doi.org/10. 1177/0269215507082741.

27. Hilton M, Pinder D. The Epley (canalith repositioning) manoeuvre for benign paroxysmal positional vertigo. Cochrane Database Syst Rev. 2004;2:CD003162.

28. Cohen HS, Kimball KT. Effectiveness of Treatments for Benign Paroxysmal Positional Vertigo of the Posterior Canal. Otol Neurotol. 2005;26(5):1034-40, https://doi. org/10.1097/01.mao.0000185044.31276.59.

29. Kulcu D, Yanik B, Boynukalin S, Kurtais Y. Efficacy of a home-based exercise program on benign paroxysmal positional vertigo compared with betahistine. J Otolaryngol Head Neck Surg. 2008;37(3):373-9.

30. Soto Varela A, Bartual Magro J, Santos Perez S, Velez Regueiro M, Lechuga Garcia R, Perez-Carro Rios A, et al. Benign paroxysmal vertigo: a comparative prospective study of the efficacy of Brandt and Daroff exercises, Semont and Epley manoevre. Rev Laryngol Otol Rhinol. 2001;122(3): 179-83.

31. Yardley L, Beech S, Weinman J. Influence of beliefs about the consequences of dizziness on handicap in people with dizziness, and the effect of therapy on beliefs. J Psychosom Res. 2001;50(1):1-6.

32. Vereeck L, Wuyts F, Truijen S, De Valck C, Van de Heyning P. The effect of early customized vestibular rehabilitation on balance after acoustic neuroma. Clin Rehabil. 2008;22(8):698713, https://doi.org/10.1177/0269215508089066.

33. Cohen HS, Kimball KT, Jenkins HA. Factors affecting recovery after acoustic neuroma resection. Acta Otolaryngol. 2002;122(8):841-50.

34. Shepard NT, Telian SA, Smith-Wheelock M, Raj A. Vestibular and balance rehabilitation therapy. Ann Otol Rhinol Laryngol. 1993;102(3 Pt 1):198-205, https://doi.org/ 10.1177/000348949310200306.

35. Hillier SL, McDonnell M. Vestibular rehabilitation for unilateral peripheral vestibular dysfunction. Cochrane 
Database Syst Rev. 2011;2, https://doi.org/10.1002/14651858. CD005397.pub3.

36. Kammerlind AC, Ledin TEA, Odkvist LM, Skargren EI. Effects of home training and additional physical therapy on recovery after acute unilateral vestibular loss - a randomized study. Clin Rehabil. 2005;19(1):54-62, https://doi. org/10.1191/0269215505cr830oa.

37. Cohen HS, Kimball KT. Increased Independence and Decreased Vertigo after Vestibular Rehabilitation. Arch Otolaryngol Head Neck Surg. 2003;128(1):60-70, https://doi. org $/ 10.1067 / \mathrm{mhn} .2003 .23$.

38. Giray M, Kirazli Y, Karapolat H, Celebisoy N, Bilgen C, Kirazli T. Short-term effects of vestibular rehabilitation in patients with chronic unilateral vestibular dysfunction: a randomized controlled study. Arch Phys Med Rehabilitation. 2009;90(8):1325-31, https://doi.org/10.1016/j.apmr. 2009.01.032.

39. Herdman SJ, Schubert MC, Das VE, Tusa RJ. Recovery of Dynamic Visual Acuity in Unilateral Vestibular Hypofunction. Arch Otolaryngol Head Neck Surg. 2003;129(8):81924, https://doi.org/10.1001/archotol.129.8.819.

40. Pavlou M, Lingeswaran A, Davies RA, Gresty MA, Bronstein AM. Simulator based rehabilitation in refractory dizziness. J Neurol. 2004;251(8):983-95, https://doi.org/10.1007/ s00415-004-0476-2.

41. Teggi R, Caldirola D, Fabiano B, Pecanati P, Bussi M. Rehabilitation after acute vestibular disorders. J Laryngol Otol. 2009;123(4):397-402, https://doi.org/10.1017/S0022215 108002983.
42. Zimbelman JE, Stoecker J, Haberkamp TJ. Outcomes in vestibular rehabilitacion. Phys Ther Case Reports. 1999; 2:232-40.

43. Szturm T, Irlandia DJ, Lessing-Turner M. Comparison of different exercise programs in the rehabilitation of patients with chronic peripheral vestibular dysfunction. J Vestib Res. 1994;4(6):461-79.

44. Mulavara AP, Richards JT, Ruttley T. Exposure to a rotating virtual environment during treadmill locomotion causes adaptation in heading direction. Exp Brain Res. 2005;166(2):210-19, https://doi.org/10.1007/s00221-0052356-0.

45. Pavlou M, Kanegaonkar RG, Swapp D, Bamiou DE, Slater M, Luxon LM. The effect of virtual reality on visual vertigo symptoms in patients with peripheral vestibular dysfunction: a pilot study. J Vestib Res. 2012;22(5-6):273-81, https:// doi.org/10.3233/VES-120462.

46. Wall C 3rd, Kentala E. Control of sway using vibrotactile feedback of body tilt in patients with moderate and severe postural control deficits. J Vestib Res. 2005;15(5-6):313-25.

47. Dozza M, Wall C, 3rd., Peterka RJ, Chiari L, Horak FB. Effects of practicing tandem gait with and without vibrotactile biofeedback in subjects with unilateral vestibularloss. J Vestib Res. 2007;17:195-204.

48. Cakrt O, Chovanec M, Funda T, Kalitová P, Betka J, Zverina E, et al. Exercise with visual feedback improves postural stability after vestibular schwannoma surgery. Eur Arch Otorhinolaryngol. 2010;267(9):1355-60, https://doi.org/10. 1007/s00405-010-1227-x.

This work is available in Open Access model and licensed under a Creative Commons Attribution-NonCommercial 3.0 Poland License - http://creativecommons.org/ licenses/by-nc/3.0/pl/deed.en. 